\title{
Diseño de estrategias de aprendizaje móvil en educación superior a través de un proceso de formación docente
}

\author{
Oscar R. Boude \\ Centro de Tecnologías Para La Academia - CTA -Universidad de La Sabana, Chía-Cundinamarca - Colombia (correo-e: \\ oscar.boude@unisabana.edu.co)
}

Recibido Sep. 20, 2020; Aceptado Nov. 16, 2020; Versión final Dic. 22, 2020, Publicado Abr. 2021

\begin{abstract}
Resumen
El objetivo de esta investigación fue fortalecer el diseño de estrategias del aprendizaje móvil en la Universidad de La Sabana (Colombia) a través de un proceso de formación docente. Se utilizó un estudio de corte cualitativo con alcance descriptivo. La muestra estuvo conformada por la totalidad de los docentes que finalizaron el proceso de formación en cada una de las cohortes ( $n 1=12$ y $n 2=7$ ). Los resultados indicaron que en la fase presencial se deben agregar ejemplos más específicos para cada disciplina y que en la fase virtual se debe ajustar la duración, pues los profesores suelen asumir nuevas responsabilidades, lo que los lleva a abandonar el proceso. Se concluye que se pueden fortalecer las estrategias de aprendizaje móvil, ya que cada uno de los docentes reconoció lo que implica el aprendizaje móvil y como integrar la estrategia de aprendizaje diseñada durante el proceso de formación.
\end{abstract}

Palabras clave: aprendizaje móvil; formación docente; tecnologías; información; comunicación; ambiente de aprendizaje mezclado

\section{Design of mobile learning strategies in higher education through a professor training process}

\begin{abstract}
The primary objective of this research study was to strengthen the design of mobile learning strategies at the University of La Sabana (Colombia) through a professor training process. This was a qualitative study with a descriptive scope. The sample included all professors who completed the training process in each of the cohorts $(n 1=12 ; n 2=7)$. The results showed that more specific examples should be added for each discipline in the face-to-face phase and that time lenght should be better adjusted in the virtual phase. This is since professors tend to take on new responsibilities, which leads to not completing and abandoning the process. It is concluded that mobile learning strategies can be strengthened given that each professor acknowledge what mobile learning implies and learnt how to integrate the learning strategies designed during the training process.
\end{abstract}

Keywords: mobile learning; professor training; information; communication technology; mixed learning environment 


\section{INTRODUCCIÓN}

Independiente de la forma en que la sociedad actual haya sido denominada por los académicos en las últimas décadas: sociedad de la información (Castells, 2011), sociedad del conocimiento (Hargreaves, 2014), sociedad del aprendizaje (Lewis, 2011) o sociedad digital (Domínguez, 2010), ésta vive un proceso de transformación que ha modificado la forma en que sus ciudadanos se comunican, trabajan, interactúan, relacionan y aprenden. Lo anterior, ha generado la necesidad de desarrollar nuevas competencias y habilidades, que permitan a los ciudadanos desempeñarse de forma adecuada y ser parte activa dentro de una sociedad dinámica y cambiante, que espera que estos puedan buscar, aprender y desaprender de manera permanente, comprender y adaptar lo aprendido a diferentes situaciones, codificar y decodificar mensajes en diferentes lenguajes audiovisuales (Area-Moreira y Pessoa, 2012).

Uno de los sectores que más ha sido afectado por estas dinámicas es la educación, pues está encargada de formar a los futuros ciudadanos de la sociedad, y más aún sobre las instituciones de educación superior quienes deben entregar a la sociedad profesionales competentes que respondan a las necesidades de las diferentes organizaciones en donde van a desempeñarse. (Romero-Rodríguez, et al., 2020). Sin embargo, para conseguirlo, éstas deberán abolir sus prácticas de silos disciplinarios, enfocados en prácticas de enseñanza esencialmente teóricas, desfragmentadas y desconectadas de los problemas reales que enfrentarán los estudiantes en su vida profesional (Han, 2014; Fung, 2017). Así como, evitar las prácticas educativas informacionales que fomentan aprendizajes repetitivos y comenzar a utilizar las tecnologías de la información y comunicación (TIC) como agentes catalizadores del proceso de aprendizaje y no como simples medios de acceso a la información.

Las universidades deben pasar de estar centradas en la transferencia del conocimiento, a convertirse en una institución de cuarta generación centrada en la solución de problemas reales. Pero en especial, los cambios que deben generarse en los diferentes procesos académicos, al respecto, Knight y Drysdale (2020) proponen que las universidades deben generar las siguientes innovaciones académicas: formular nuevas modalidades de formación a la carta, expedición de nuevas certificaciones, nuevos modelos de apoyo a los estudiantes, innovaciones curriculares, nuevas prácticas pedagógicas, nuevos roles docentes y nuevas estrategias de evaluación. Sin embargo, ninguna de estas modificaciones sería posible en la institución educativa, si no existe un cambio de percepción del papel que juega el profesor universitario en este proceso, ya que es, a través de la práctica docente que estas innovaciones se desarrollan en el aula de clase.

En la actualidad, son muchos los profesores que han iniciado el proceso de transformación de sus prácticas docentes a través de la integración de algún tipo de tecnología educativa, con el fin de que estas respondan a las expectativas y necesidades de sus estudiantes. Para hacerlo, algunos de ellos suelen recurrir a blogs, redes académicas, portales educativos o redes sociales, buscando experiencias exitosas que han sido reportadas por otros docentes (Boude, 2019), lo cual estaría bien, si estos modificaran sus prácticas con estas experiencias a través de un proceso de reflexión sobre su quehacer docente, pero suelen ir a estos sitios en búsqueda de la herramienta que solucione todo, y sin pensarlo terminan generando tensiones en el proceso de formación, pues los resultados no son tan exitosos como fueron reportados por sus colegas. Más aún, muchas de estas tensiones ocurren por la brecha generacional que existe entre docentes y dicentes, debido a que cada uno de ellos tiene una concepción diferente de cómo debería desarrollarse el proceso de formación a través de la mediación de las TIC.

Incluso cuando se trata de integrar una tecnología común a ambos como lo son los dispositivos móviles, debido a que cada uno de ellos tiene una concepción diferente de cómo utilizarlos en el aula. Para integrar dispositivos móviles en el proceso de formación, se debe partir del reconocimiento de lo que es el Aprendizaje Móvil (AM), comprender el contexto en donde se abordará, las competencias a desarrollar y las necesidades educativas y expectativas de quienes lo utilizarán (Boude y Jiménez,2015). Por lo anterior, si se quieren fortalecer los procesos de formación de los estudiantes, a través de la integración de dispositivos móviles, primero es necesario formar a los docentes de la institución, para que estos reconozcan lo que es el AM y a partir de allí, junto con los resultados esperados de aprendizaje y las características de la población, puedan formular las estrategias de aprendizaje móvil (Pratama y Scarlatos, 2020). Al respecto, este trabajo muestra los resultados obtenidos en un proyecto realizado en la Universidad de La Sabana, cuya finalidad fue fortalecer la integración del AM en la institución.

\section{OTROS ANTECEDENTES}

Es importante mencionar que el concepto de dispositivo móvil ha ido cambiando a lo largo del tiempo, acorde con los avances que han tenido las ciencias de la Computación y la Electrónica. Inicialmente el concepto estaba asociado a la capacidad de abordar múltiples contenidos en diversos momentos o lugares a través de Asistentes digitales personales, portátiles o computadores de escritorio (Sarrab, et al., 2012), después su 
concepción estaba orientada hacia la portabilidad del aparato y su capacidad intermitente de conexión a Internet (Cheon, et al., 2012), en la actualidad, el concepto gira entorno a que son aparatos pequeños, diseñados para una función principal pero que pueden cumplir otras funciones y cuentan con conectividad permanente a Internet (Al-Hamad, et al., 2020), bajo esta definición caben las Playstation portables, los reproductores de audio y video, los navegadores GPS, los teléfonos móviles, los smartphones, las tabletas, entre otros.

Sin embargo, resulta aún más relevante comprender las concepciones teóricas que hay alrededor del AM, al respecto, Caudill, (2007) indica que el AM es una extensión del E-learning, debido a que el dispositivo móvil permite acceder al proceso formativo en cualquier momento o lugar. Por su parte, Kearney et al. (2012) y Vavoula y Sharples (2009) plantean que el AM es equivalente al E-learning apoyado en dispositivos móviles, así mismo, existen otras concepciones centradas en el proceso de aprendizaje que proponen, diseñar estrategias de AM a partir del reconocimiento de sus fortalezas y limitaciones (Rezaee, et al.,2020) así como, de los aprendizajes que el docente espera fortalecer en sus estudiantes (Sung y Mayer, 2013; Danish y HmeloSilver, 2020; Mayer, 2020). Para realizar el proceso de formación se tomó como referencia el modelo propuesto por Boude y Jiménez (2015). El cuál propone que para diseñar estrategias de aprendizaje móvil se debe pasar por cinco fases diferentes denominadas: Reconocimiento, Análisis, Fundamentación, Diseño e Implementación, como puede observarse en la figura 1.

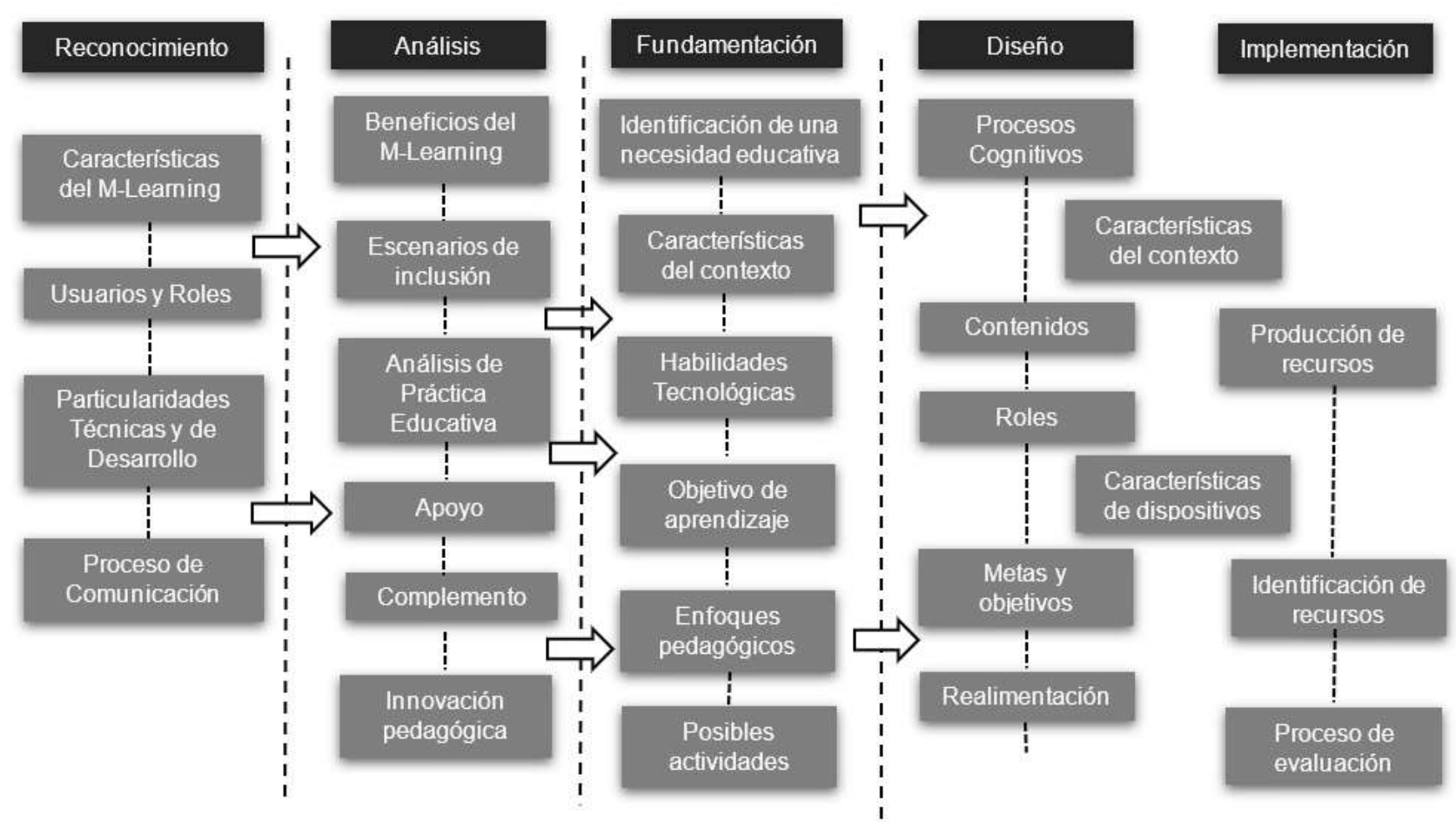

Fig. 1: Modelo para el diseño de estrategias de aprendizaje móvil (Tomado, con autorización, de Boude y Jiménez, 2015)

\section{METODOLOGÍA}

El enfoque metodológico utilizado fue cualitativo con un alcance descriptivo, la muestra estuvo conformada por los 19 docentes que finalizaron los dos procesos de formación que se realizaron durante el proyecto. La primera cohorte $\mathrm{n} 1=12$ y la segunda $\mathrm{n} 2=7$. Ahora bien, cabe anotar que este proyecto lo iniciaron 37 profesores, y aunque lo terminaron solo el $51 \%$ de los docentes, la muestra contó con al menos un docente de la mayoría de las facultades de la Universidad, entre las que están: Medicina, Enfermería y Rehabilitación, Comunicación Social, Ingeniería, Psicología, la Escuela de Negocios, Derecho y Educación.

El estudio fue dividido en tres fases, la primera de ellas estuvo orientada a diseñar el ambiente de aprendizaje mezclado en el que participaron los docentes, para esto se utilizó el modelo antes mencionado que consta de cinco fases denominadas: Reconocimiento, Análisis, Fundamentación, Diseño e Implementación. En la segunda fase, se realizó el proceso de formación docente durante los periodos inter-semestrales, dicho proceso estuvo dividido en dos componentes como se muestra en la figura 2. El componente presencial tuvo la finalidad de dar la fundamentación conceptual y el desarrollo de habilidades en el diseño de estrategias de aprendizaje móvil y el componente virtual estuvo orientado al acompañamiento de los profesores en el diseño final de la estrategia de AM a proponer. 


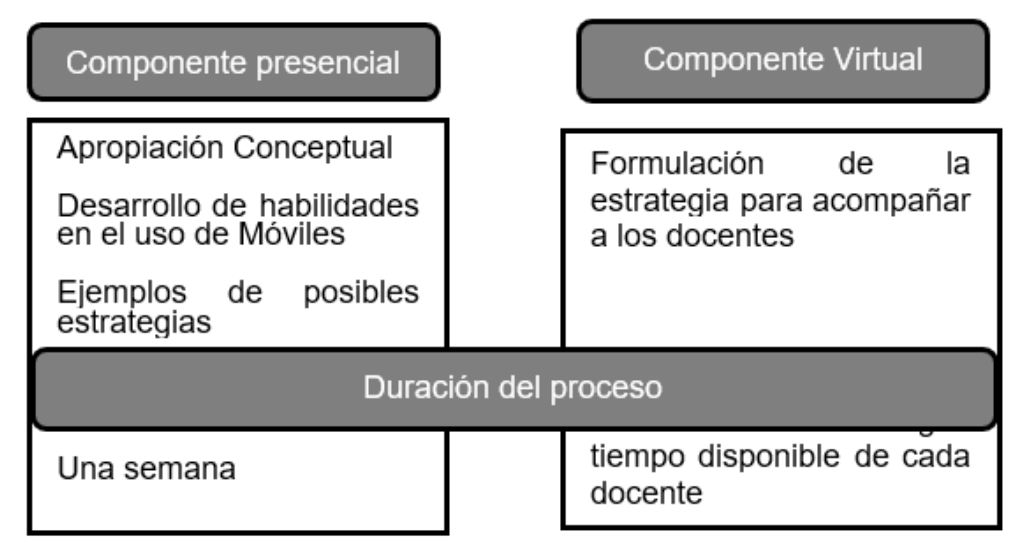

Fig. 2: Ambiente de aprendizaje diseñado

Asimismo, se realizó la recolección de los datos a través de observaciones no participantes durante el proceso de formación presencial, las estrategias de AM formuladas por los docentes y una entrevista semiestructurada, diseñada para identificar la percepción que tuvieron los docentes del proceso de formación. Además, se realizó un grupo focal con los docentes que no terminaron el proceso de formación, para identificar las razones por las cuales abandonaron el proceso. Los instrumentos utilizados para la entrevista, el grupo focal y la formulación de la estrategia de AM dentro del estudio, fueron validados a través de juicios de expertos antes de ser aplicados.

En la tercera y última fase se realizó el análisis de los datos recolectados, la información fue primero segmentada y categorizada, producto de este proceso se definieron las siguientes categorías: habilidades desarrolladas, dificultades encontradas y estrategias diseñadas. Las cuales fueron utilizadas para realizar la triangulación de los datos, con el fin de identificar las contribuciones del proceso de formación en el diseño de estrategias de AM por parte de los docentes de la institución. Para realizar este proceso se utilizó el software de análisis de datos cualitativos Atlas ti.

\section{RESULTADOS Y DISCUSIÓN}

Como se mencionó en el apartado metodológico, la finalidad del estudio estuvo enfocada en identificar los aportes del proceso de formación al diseño de estrategias de AM, así como las estrategias diseñadas. Por tal razón, los resultados se mostrarán en ese orden. A nivel de los hallazgos encontrados, lo primero que se pude indicar es que todos los docentes que hicieron parte del proceso de formación en la modalidad presencial culminaron con éxito esta fase, reconociendo lo que es el AM, así como, aquellas situaciones en donde es más adecuado integrar los dispositivos móviles como agentes catalizadores del proceso de formación. Sin embargo, tras finalizar las seis semanas del proceso de formación virtual en ambas cohortes se presentó una deserción del $37 \%$ y $47 \%$ respectivamente, debido a la falta de tiempo de los docentes para continuar el proceso, nuevos encargos durante el proceso intersemestral y a desinterés en la temática.

En efecto, al indagar más con los participantes alrededor de lo sucedido en cada una de las fases del proceso de formación, fue posible identificar varias dificultades que ayudan a comprender las tasas de deserción y que se debe de tener en cuenta para el desarrollo de los procesos de formación docente. A nivel del componente presencial del proceso de formación los participantes indicaron que las principales dificultades estaban en: a) El tiempo destinado a la conceptualización de la temática, sugirieron dedicar al menos un $40 \%$ más de tiempo a este componente; b) La formulación de ejemplos particulares para cada área disciplinar, que les permitiera identificar como integrar los dispositivos en sus procesos formativos específicos; y c) Establecer proceso de tutoría personalizada, esto debido a la diversidad de conocimientos con los que contaban los profesores participantes en el estudio, pues para algunos era la primera vez que escuchaban del aprendizaje móvil

A nivel del componente virtual del proceso de formación los participantes indicaron: a) Incluir más recursos como videos en donde se observase a docentes integrando la estrategia de AM; b) Modificar las guías utilizadas para la formulación de la estrategia de AM, ya que se repiten muchos componentes dentro de ellas y esto genera confusión en los docentes; y c) Disminuir la duración del componente de formación virtual, debido a que se confían del tiempo que tienen y coordinan otras actividades, las cuales al final terminan impidiendo la formulación de la estrategia. 
Es importante resaltar que a diferencia de lo que se encuentra en la literatura en donde la mayoría de las experiencias de AM se encuentran en las facultades de Ingeniería y del Área de la Salud, en este estudio la mayoría de las propuestas pertenecían a las facultades de Comunicación, Educación, Enfermería e Ingeniería. A continuación, se indican algunas de las estrategias de AM formuladas por los docentes durante este proceso, es preciso mencionar que se asignaron títulos a las propuestas de los profesores para que sea más sencillo de identificar sus usos en educación superior.

\section{Historias digitales con celumetrajes}

La propuesta presentada está formulada para estudiantes de comunicación social o áreas afines después de tercer semestre, con la finalidad de que estos puedan generar un pequeño celumetraje de dos a tres minutos para contar una historia. Para esto, el profesor dividió el curso en equipos de tres estudiantes, dándole a cada grupo una temática a utilizar en la historia. Debido a que la producción completa del celumetraje debería realizarse en las dos sesiones programadas, el docente envío a los estudiantes con anticipación la temática que iban a abordar, para que estos realizarán la producción de los guiones antes de la clase y utilizar el tiempo de las sesiones en grabar las diferentes escenas con ayuda de los dispositivos móviles y editar las tomas finales en los computadores del aula de clases. Al finalizar el proceso, los celumetrajes fueron presentados por cada equipo y se realizó el proceso de co-evaluación por parte de los demás grupos utilizando una rúbrica diseñada por el docente para tal fin.

Aunque esta estrategia fue planificada para ser utilizada por estudiantes con cierto nivel de conocimiento de edición de video, esta puede ser usada por cualquier área de formación, hoy existen muchas aplicaciones disponibles en línea en donde se pueden editar clips de video fácilmente, además, una de las mejores formas que existen para identificar los aprendizajes de los estudiantes y si estos son competentes, es solicitarles que generen productos de conocimiento en donde deban transformar el conocimiento en nuevas formas de representación del mismo.

\section{Generación de productos digitales}

La estrategia de AM fue formulada por un docente de la facultad de Educación, quien propuso el uso de los dispositivos móviles para que los estudiantes que estaban en prácticas generaran contenidos educativos para los estudiantes a los cuales les estaban enseñando. La finalidad de esta actividad tuvo un doble propósito, favorecer el desarrollo de las competencias alrededor de la asignatura de práctica pedagógica y la elaboración de recursos educativos digitales que los alumnos pudieran utilizar en sus prácticas profesionales.

Los estudiantes estaban divididos en grupos de 4 personas, estos debían acordar la temática que iban a abordar y como esta podría ayudar a las actividades que tenían planificadas dentro de su práctica. Todo el proceso de formulación del producto digital se hacía a través del trabajo independiente recibiendo realimentación de su profesor, así mismo, se apropiaban de las herramientas necesarias para desarrollar el producto a través de tutoriales en Youtube, el docente se reunía semanalmente con los estudiantes para validar los avances del proceso. Al igual que la estrategia presentada previamente, esta se puede utilizar en diferentes áreas, si bien los alumnos no están en sus prácticas formando a otras personas, si pueden desarrollar con los dispositivos, productos digitales de conocimiento utilizando sus propios dispositivos móviles.

\section{De aprendiz a formador}

La estrategia, estuvo dirigida a estudiantes de enfermería de quinto semestre quienes están encargados de formar a las madres que acaban de dar a luz a sus hijos, este es un proceso por el que tanto las mamás como los estudiantes de enfermería deben pasar y tiene como finalidad enseñarles a éstas, sobre todo a las primerizas, elementos básicos en el cuidado materno, así como en las alertas que debe tener cuando este con su pequeño hijo en casa. Sin embargo, en muchas ocasiones la forma y los tiempos con los que se cuenta en el hospital no son los suficientes para garantizar que la madre ha logrado desarrollar las habilidades necesarias en el cuidado de su hijo y cuando llegan a casa y no cuenta con el apoyo del personal de enfermería las preguntas suelen aparecer.

Por esta razón, la estrategia buscó que los estudiantes desarrollaran una aplicación móvil que brindará a los padres las respuestas a la mayoría de los interrogantes que suelen tener luego de regresar a casa. Los estudiantes en equipos planificaron, diseñaron y crearon la app con las orientaciones de su docente. La actividad buscó que los estudiantes reflexionaran sobre la importancia de su proceso de formación y lograran comprender las interrogantes reales que tenían los padres al volver a casa y como estos vacíos podrían llenarse transformando sus estrategias de formación. 


\section{Asumiendo el rol de un profesional}

La estrategia, no pretendió fortalecer una competencia específica, por el contrario, estuvo orientada a aquellos estudiantes que aún no formaban parte del programa de Ingeniería Química, pero que buscaban tener una experiencia cercana sobre lo que implicaba pertenecer a esta carrera. Los usuarios de esta estrategia fueron los estudiantes de último grado de educación media que asistían al Open Campus que es organizado por la Universidad cada semestre y que ofrece a los visitantes la oportunidad de vivir la experiencia de saber los procesos que son desarrollados por los diferentes programas académicos con los que cuenta la Universidad de La Sabana.

La finalidad de esta estrategia estaba orientada a brindar a los visitantes una serie de problemas reales que han sido solucionados por estudiantes de Ingeniería Química. Para lo cual, los participantes en una primera instancia conformaron equipos de 5 personas, a los cuales se les entregó una tableta de 7 pulgadas que contenía la aplicación de realidad aumentada Aurasma, junto con el acceso a un sitio web en donde estaban los casos que debían solucionar. Durante el proceso los participantes contaban con el apoyo de consultores, los docentes de la facultad, quienes apoyaban a los equipos en la toma decisiones y les orientaban sobre los procesos que debían seguir para lograr resolver los casos en el menor tiempo posible.

Es importante recordar que la finalidad de la estrategia era lograr que los futuros estudiantes de la carrera se sintieran parte de un proceso real, por lo que durante el proceso los profesores y estudiantes de la carrera que participaron de esta experiencia siempre asumieron sus roles como consultores y personal de apoyo a los equipos participantes. Este tipo de estrategia brinda al profesor la posibilidad de integrar la solución de problemas reales al proceso formativo de sus estudiantes, así como, la capacidad de asumir diferentes roles, tales como: consultor, asesor, experto temático, entre otros.

\section{DISCUSIÓN}

Desde hace más de una década se está insistiendo en la pertinencia de integrar los dispositivos móviles a los procesos de formación (Caudill, 2007; Cheon et al., 2012; Vavuola et al., 2009), sin embargo, la realidad es que las instituciones de educación superior, lejos están de utilizar estos artefactos como un agente catalizador del proceso de aprendizaje y su uso está más sujeto a verlos como herramientas de acceso a plataformas, bibliotecas o sistemas de gestión de aprendizajes -LMS-. (Brennan, 2015)

Lo anterior se debe en parte a la falta de planes concretos por parte de las instituciones educativas de educación superior para fomentar la integración de este tipo de artefactos, así como, al desconocimiento de los profesores de las potencialidades del AM y a la carga académica con las que cuentan los docentes, quienes necesitan de tiempo para poder diseñar e implementar las estrategias de aprendizaje móvil. Como ocurrió en este proyecto en donde una de las principales razones expuestas por los docentes para no finalizar el proceso, fue la falta de tiempo y cargas académicas. (Lai y Wu, 2016; Kuhnel, et al., 2018)

Asimismo, resulta fundamental seguir trabajando en mejorar las competencias digitales de los docentes de educación superior, pues por primera vez en la historia las habilidades tecnológicas que tienen los estudiantes son muy superiores a la poseen la mayoría de sus docentes, lo cual genera fricciones en el proceso educativo, gracias a la diferencia que existe entre las concepciones que tienen dicentes y docentes, sobre la forma en que deberían integrarse las tecnologías al proceso educativo y el papel que debe jugar el profesor en éste proceso (Ramírez-Montoya y García-Peñalvo.2017).

Debido a esto, algunos docentes universitarios han ingresado en una carrera por transformar sus prácticas pedagógicas a través de la incorporación de diversas tecnologías en el aula, sin embargo, como ha sido demostrado en la reciente situación mundial a causa de la pandemia, la mayoría de estos no cuentan con las competencias digitales para hacerlo, ni con el conocimiento pedagógico para realizarlo, pues la mayoría de ellos son profesionales que hacen parte del proceso de formación universitario a través de la modalidad de profesores de hora cátedra.

En Colombia, los docentes con los niveles más bajos en el desarrollo de las competencias digitales, suelen ser los pertenecientes al área de las Ciencias Sociales (Osorio y Alvarado, 2019), No obstante, en este trabajo se logró evidenciar que esto es posible de superar si se realiza un proceso personalizado de acompañamiento, en el cual el docente logre cambiar la concepción que tiene alrededor del papel que juegan las TIC en el proceso de formación de sus estudiantes y se dé cuenta de cómo éstas pueden potenciar su práctica docente sin sacrificar sus concepciones pedagógicas.

Además, cuando la tecnología que se va a incorporar son los dispositivos móviles, debido a que éstos son una de las pocas herramientas tecnológicas que permiten que el proceso de aprendizaje se de en todo 
momento y lugar (Al-Hamad, et al., 2020; Cheon et al., 2012 ), así como, un amplió registro de información de un momento, lugar o fenómeno específico a estudiar (Bernacki, et al., 2020; Sidik y Syafar, 2020), lo cual, resulta fundamental a la hora de comprender la complejidad de los procesos sociales.

Para finalizar, es importante destacar que después de que los docentes son conscientes de lo que implica el aprendizaje móvil, sus ventajas y limitaciones, éstos no solo son capaces de formular diversas estrategias que contribuyen a fortalecer los aprendizajes de sus estudiantes, sino también, logran transformar sus roles dentro del aula, pasando de ser el centro del proceso formativo, a agentes catalizadores del mismo a través de la orientación y acompañamiento de los estudiantes.

\section{CONCLUSIONES}

La finalidad de este proyecto fue la de fortalecer el desarrollo de estrategias de AM en los profesores de las diferentes facultades de la Universidad de La Sabana, a través de un proceso de formación mezclado. Lo cual se logró debido a que al finalizar el proceso al menos uno de los profesores de la mayoría de las facultades diseñó una estrategia para fortalecer sus prácticas docentes a través de los dispositivos móviles.

No obstante, se deben modificar los tiempos en el componente virtual, pues esto resulta perjudicial para el proceso debido a que los profesores se concentran en sus actividades académicas y descuidan el proceso de formación, llevándolos a la deserción del proceso de formación.

Sin embargo, aquello docentes que terminan el proceso y logran comprender lo que implica la integración de los dispositivos, formulan estrategias de AM que logran innovar sus procesos de formación y transformar su visión alrededor del cuál debe ser su rol en el proceso.

Asimismo, resulta relevante que las intuiciones de educación superior cuenten con políticas claras alrededor de la incorporación de los dispositivos móviles a los procesos de formación, ya que durante el proceso de formación varios docentes desconocían si estaba permitido hacer uso de los dispositivos móviles de los estudiantes, o si existía alguna directriz institucional para integrar estos dispositivos en los procesos de formación.

En el mismo sentido, es importante que las instituciones de educación superior tomen conciencia de la importancia que tiene la integración de dispositivos móviles como agentes catalizadores del proceso de formación de los estudiantes. Esto porque no solo es la única tecnología común entre docentes y discentes, sino también, es una de las pocas que permite al docente utilizar los elementos del contexto físico en donde están ubicados los estudiantes, como parte activa del proceso de formación. Así un profesor de comunicación social puede orientar a su estudiante durante el desarrollo de una noticia que se le ha solicitado cubrir.

\section{REFERENCIAS}

Alemán, I., Vera, E., y Patiño-Torres, M. J., COVID-19 and medical education: Challenges and opportunities in Venezuela [COVID-19 y la educación médica: retos y oportunidades en Venezuela], doi: 10.1016/j.edumed.2020.06.005, Educación Médica (2020).

Al-Hamad, N. Q., AlHamad, A. Q., y Al-Omari, F. A., Smart devices employment in teaching and learning: reality and challenges in Jordan universities, doi: 10.1186/s40561-020-0115-0, Smart Learning Environments, 7(1), 1-15 (2020).

Area-Moreira, M., y Pessoa, T., De lo sólido a lo líquido: las nuevas alfabetizaciones ante los cambios culturales de la Web 2.0, doi: 10.3916/C38-2011-02-0, Comunicar, 19(38), 13-20 (2012).

Boude, O., Como integran los docentes los dispositivos móviles en el aula. Revista ESPACIOS, 40(29) (2019).

Boude, O., y Jiménez, J., Framework for design of mobile learning strategies. Handbook of Mobile Teaching and Learning, 1-13 (2015).

Bernacki, M. L., Greene, J. A., y Crompton, H., Mobile technology, learning, and achievement: Advances in understanding and measuring the role of mobile technology in education. Contemporary Educational Psychology, 60, 101827 (2020).

Brennan, K., Beyond technocentrism, Constructivist Foundations, 10 (3), 289-296 (2015).

Castells, M. Network theory| A network theory of power. International journal of communication, 5, 15 (2011).

Caudill, J. G., The growth of m-learning and the growth of mobile computing: Parallel developments, doi: 10.19173/irrodl.v8i2.348, The International Review of Research in Open and Distributed Learning, 8 (2), 1-13 (2007).

Cheon, J., Lee, S., Crooks, S. M., y Song, J., An investigation of mobile learning readiness in higher education based on the theory of planned behavior, doi: 10.1016/j.compedu.2012.04.015, Computers y education, 59 (3), $1054-1064$ (2012).

Danish, J., y Hmelo-Silver, C. E., On activities and affordances for mobile learning, doi: 10.1016/j.cedpsych.2019.10182, 9Contemporary Educational Psychology, 60, 101829 (2020). 
Fung, D., A connected Curriculum for Higher Education, 1ª edición, UCL Press, Londres, Inglaterra (2017).

García, L., Bases, mediaciones y futuro de la educación a distancia en la sociedad digital. 1aㅡ edición, Editorial Síntesis, Madrid, España (2014).

Han, B. C., En el enjambre. Barcelona, 1ª edición, Herder Editorial, España (2014).

Hargreaves, D. H. A self-improving school system and its potential for reducing inequality. Oxford Review of Education, 40(6), 696-714 (2014).

Kearney, M., Schuck, S., Burden, K., y Aubusson, P., Viewing mobile learning from a pedagogical perspective. Alt-JResearch In Learning Technology, 20(1) (2012).

Knight, G. L., y Drysdale, T. D., The future of higher education (HE) hangs on innovating our assessment-but are we ready, willing and able?. Higher Education Pedagogies, 5(1), 57-60 (2020).

Kuhnel, M., Seiler, L., Honal, A., y Ifenthaler, D., Mobile learning analytics in higher education: Usability testing and evaluation of an app prototype, doi: 10.1108/ITSE-04-2018-0024, Interactive Technology and Smart Education, 15 (4), 332-347, (2018).

Lai, C.Y., y Wu, C.C., Promoting nursing students' clinical learning through a mobile e-portfolio, doi: 10.1097/CIN.0000000000000263, CIN: Computers, Informatics, Nursing, 34(11), 535-543 (2016).

Lewis, T. E. Rethinking the learning society: Giorgio Agamben on studying, stupidity, and impotence. Studies in Philosophy and Education, 30(6), 585-599 (2011).

Mayer, R. E., Where is the learning in mobile technologies for learning?, doi: 10.1016/j.cedpsych.2019.101824, Contemporary Educational Psychology, 60, 101824, (2020)

Osorio, L. M. C., y Alvarado, M. F. C., Reflexiones en torno a la necesidad de cultivar competencias tecnológicas en docentes de educación superior desde una perspectiva pedagógica. Revista Colombiana de Computación, 20(2), 28-36 (2019).

Pratama, A. R., y Scarlatos, L. L. The roles of device ownership and infrastructure in promoting E-learning and M-learning in Indonesia. International Journal of Mobile and Blended Learning (IJMBL), 12(4), 1-16 (2020).

Ramírez-Montoya, M. S., y García-Peñalvo, F. J. La integración efectiva del dispositivo móvil en la educación y en el aprendizaje. Revista Iberoamericana de Educación a Distancia, doi:10.5944/ried.20.2.18884, 20(2), 29-47 (2017).

Rezaee, A. A., Alavi, S. M., y Razzaghifard, P. Mobile-based dynamic assessment and the development of EFL students' oral fluency. International Journal of Mobile Learning and Organisation, 14(4), 511-532 (2020).

Romero-Rodríguez, J. M., Aznar-Díaz, I., Hinojo-Lucena, F. J., y Cáceres-Reche, M. P., Models of good teaching practices for mobile learning in higher education, doi: 10.1057/s41599-020-0468-6, Palgrave Communications, 6 (1), 1-7 (2020).

Sarrab, M., Elgamel, L., y Aldabbas, H., Mobile learning (m-learning) and educational environments, doi: 10.5121/ijdps.2012.3404, International journal of distributed and parallel systems, 3(4), 31 (2012).

Sidik, D., y Syafar, F. Exploring the factors influencing student's intention to use mobile learning in Indonesia higher education. Education and Information Technologies, 25(6), 4781-4796 (2020).

Sung, E., y Mayer, R. E., Online multimedia learning with mobile devices and desktop computers: An experimental test of Clark's methods-not-medxia hypothesis, doi: 10.1016/j.chb.2012.10.022, Computers in Human Behavior, 29(3), 639-647 (2013).

Vavoula, G., y Sharples, M., Meeting the challenges in evaluating mobile learning: A 3-level evaluation framework, International Journal of Mobile and Blended Learning (IJMBL), 1(2), 54-75 (2009). 